DOI: 10.46340/eujem.2020.6.5.13

Tetiana Yatsyk

ORCID ID: https://orcid.org/0000-0002-7294-5973

Taras Shevchenko National University of Kyiv, Ukraine

\title{
A MULTIPLE LINEAR REGRESSION MODEL FOR CRYPTOCURRENCY PRICE IN THE FINANCIAL ANALYSIS AND ACCOUNTING
}

The cryptocurrency market is represented by more than 6,099 different cryptocurrencies with a total market capitalization of USD 354,316 million with Bitcoin dominance over $60 \%$. Despite the increasing amount of scientific research, a comprehensive analysis of factors influencing the price of cryptocurrency is still needed. Previous studies have focused on the Bitcoin capitalization changes, rather than relationships and dependencies between the price of different cryptocurrencies and other factors. The author proposed a multiple linear regression model, which can be used for the cryptocurrency price forecast. The author tested the hypothesis, that Bitcoin's closing price changes likely in response to changes in altcoin prices and Google search index as well. According to the conducted research, the price of Bitcoin depends significantly on Google's search index on the specific cryptocurrency name. The revealed multiple regression equation can be further used for creating operational analytical programs for forecasting the price movement of Bitcoin.

Keywords: cryptocurrencies, cryptoassets, distributed ledger technology (DLT), cryptocurrency financial analysis, multiple linear regression model, regression model.

Introduction. The beginning of 2020 was quite stressful for most cryptocurrency holders, especially for crypto exchanges and brokers. Thus, on January 10, 2020, the fifth Anti Money Laundering (AML) Directive took effect. Some cryptocurrency platforms announced their closure; others have to struggle to meet the new requirements. There is no single agreed definition of cryptoassets, but generally, cryptoassets are a cryptographically secured digital representation of value or contractual rights that is powered by forms of DLT and can be stored, transferred or traded electronically ${ }^{1}$. Most authors categorized cryptoassets into three types of tokens: exchange tokens, security tokens, utility tokens ${ }^{2}$. While cryptocurrency could be an example of exchange tokens that are typically used as a means of exchange, usually functioning as a decentralized tool to enable the buying and selling of goods and services, or to facilitate regulated payment services. Exchange tokens are not issued or backed by any central authority and are intended and designed to be used as a means of exchange. They are, usually, a decentralized tool for buying and selling goods and services without traditional intermediaries.

As long as cryptoassets can be used as a means of exchange, they are not considered a currency or money, as both the Bank of England and the G20 Finance Ministers and Central Bank Governors have previously set out. They are too volatile to be a good store of value, they are not widely accepted as a means of exchange, and they are not used as a unit of account.

According to the research conducted by coinmarketcap.com, there were more than 6,099 different cryptocurrencies with total market capitalization of USD 354,316 million and Bitcoin dominance over 60\% (table 1$)^{3}$. The distinguishing factors of all these cryptocurrencies are a development route, consensus algorithm, governance, use of "Smart contracts", exchange rate control, acceptability, anonymity and privacy,

\footnotetext{
${ }^{1}$ EY (2019). IFRS Developments: Holdings of cryptocurrencies < https://www.ey.com/Publication/vwLUAssets/ ey-devel150-cryptocurrency-holdings-august-2019/USDFILE/ey-devel150-cryptocurrency-holdings-august-2019.pdf> (2020, August, 25).

${ }^{2}$ Ankenbrand, T., Bieri, D., Cortivo, R., Hoehener, J., Hardjono T. (2020). Proposal for a Comprehensive (Crypto) Asset Taxonomy. Crypto Valley Conference on Blockchain Technology (CVCBT), Rotkreuz, Switzerland, 16-26. ${ }^{3}$ Coinmarketcap (2020). Cryptocurrencies market capitalization <https://coinmarketcap.com/currencies/bitcoin/> (2020, August, 25). [in English].
} 
permissions and other factors. It is important to note that Bitcoin has not actually dropped as far as global markets have in this first, tumultuous quarter of 2020 . The cryptoassets only dropped by $12 \%$.

Altcoins are all coins that are an alternative to Bitcoin. There are two types of altcoins: those that built using Bitcoin's original open-source protocol, with a number of changes to its underlying codes, conceiving a new coin with a different set of features; altcoins that are not based on Bitcoin's open-source protocol, but that have their own protocol and distributed ledger (Ethereum and Ripple).

Table 1

TOP 10 cryptocurrencies by market capitalization in August 2020

\begin{tabular}{ccccc}
\hline$\#$ & Name & Market Cap, USD million & Unit Price, USD & Change (24h), \% \\
\hline 1 & Bitcoin & 215,617 & 11,685 & $3.90 \%$ \\
2 & Ethereum & 44,905 & 401 & $3.19 \%$ \\
3 & XRP & 13,728 & 0.3 & $2.23 \%$ \\
4 & Tether & 10,037 & 1 & $0.31 \%$ \\
5 & Bitcoin Cash & 5,471 & 296 & $2.85 \%$ \\
6 & Bitcoin SV & 4,339 & 235 & $4.66 \%$ \\
7 & Litecoin & 3,871 & 59 & $3.36 \%$ \\
8 & Cardano & 3,733 & 0.1 & $-0.08 \%$ \\
9 & Binance Coin & 3,366 & 23 & $6.09 \%$ \\
10 & Link Platform & 3,354 & 10 & $0.99 \%$ \\
& Other & 45,896 & - & - \\
\hline
\end{tabular}

Source: Compiled by the author based on data from $^{l}$.

Exchange tokens (like Bitcoin, Litecoin etc.) are not issued or backed by any central authority and can be used directly as a means of exchange. These tokens can enable the buying and selling of goods and services without the need for traditional intermediaries such as central or commercial banks ${ }^{2}$.

Many scientists have been engaged in solving the problem of cryptocurrency forecasting, however the majority of research focused mostly on Bitcoin capitalization, rather than on a diverse taxonomy of these new digital assets. While most scientists agree, that external factors have considerable effect on cryptocurrencies, there are still very little research on the level of dependence between the prices of Bitcoin and other altcoins. The author performed an analysis of different cryptocurrencies' life cycles in their economic environment, followed by a multiple linear regression model for cryptocurrency price forecast in the financial analysis and accounting.

The objective of this paper is to attempt to build a multiple linear regression model, which can be used for cryptocurrency price forecast and to understand the relationship between cryptocurrency price and other analyzed variables. While most scientists agree, that external factors have considerable effect on cryptocurrencies, there are still very little research on the level of dependence between the prices of Bitcoin and other altcoins.

Literature review. Despite the increasing amount of the scientific research, a comprehensive analysis of factors influencing the price of cryptocurrency is still needed. Previous studies have focused on the Bitcoin capitalization changes, rather than relationships and dependencies between the price of different cryptocurrencies and other factors.

Akbulaev et al. (2020) analyzed existing literature on the basic characteristics of main cryptocurrencies and performed the analysis of the relationship between Bitcoin and Ethereum. The authors presented a mathematical model of the cryptocurrency relationship ${ }^{3}$.

\footnotetext{
${ }^{1}$ Coindesk (2020). Bitcoin market capitalization <https://www.coindesk.com/price/bitcoin> (2020, August, 25).

${ }^{2}$ Financial Conduct Authority (FCA) (2019). Guidance on Cryptoassets. Consultation Paper CP19/3 $<$ https://www.fca.org.uk/publication/consultation/cp19-03.pdf> (2020, September 17).

${ }^{3}$ Akbulaev, N., Mammadov, I., Hemdullayeva, M. (2020). Correlation and Regression Analysis of the Relation between Ethereum Price and Both Its Volume and Bitcoin Price. The Journal of Structured Finance.
}

DOI: jsf.2020.1.099. 10.3905/jsf.2020.1.099. 
Teker, Dilek \& Deniz (2020) defined the main factors that influence significantly the cryptocurrency prices, among which external macroeconomic factors ${ }^{1}$.

Brukhanskyi \& Spilnyk (2019) identified and justified the prospects for solving the problem of integrating new cryptographic objects into the accounting and reporting system. The authors highlighted the need to generate the accounting status of cryptoassets ${ }^{2}$.

Bouri et al. (2018) examined the nonlinear, asymmetric and quantile effects of aggregate commodity index and gold prices on the price of Bitcoin using several advanced autoregressive distributed lag (ARDL) models. The authors indicated the possibility to forecast Bitcoin price movements based on price information from the aggregate commodity index and gold prices ${ }^{3}$.

Numerous studies have already been conducted in Ukraine to forecast cryptocurrency prices using a machine-learning model. Tarasova, et al. (2020) determined the basic features of the use of mathematical modeling of the system to forecast cryptocurrency exchange rate ${ }^{4}$. The authors specified advantages and disadvantages of different cryptocurrency. Yatsyk (2018) studied the taxonomy of cryptoassets and methodology of the financial accounting of cryptocurrencies according to the international financial reporting standards (IFRS) ${ }^{5}$.

Methods. In a comprehensive overview of cryptocurrency market environment, this paper uses statistical methods, as well as dynamic and comparative analysis. A modern regression model was firstly introduced by Fisher $(1925)^{6}$, he modified the regression theory of Pearson (1926) and Yule (1907) ${ }^{7}$ and the least squares theory of Gauss $(1963)^{8}$. Fisher firstly realized that the distribution associated with the regression coefficient was unaffected by the distribution of X. Subsequently Fisher interpreted the fixed X assumption in terms of his notion of ancillarity. The basic principles of modern regression model were used in this paper. To estimate a multiple regression model and relationship between cryptocurrency price and other variables statistical analysis was performed using EViews software. The selected sample includes daily data of cryptocurrency closing price, market capitalization, Google's search index. The price of Bitcoin was analyzed as dependent variable on the prices of other altcoins and Google's search index. Data was gathered from multiple sources at various time points during December 2013 - January 2020.

A multiple linear regression is a method to model a relationship between a dependent variable and a number of independent variables that could be described by the following formula (1):

$$
Y=X+e
$$

The author proposed the multiple regression model and estimated its parameters by the method of Ordinary Least Squares (OLS). To explain the proportion of the variance in the dependent variable that is predictable from the independent variable the author used the coefficient of determination:

$$
R^{2}=\frac{\operatorname{Var}\left(X^{\hat{1}}\right)}{\operatorname{Var}(Y)}=1 \quad \frac{\operatorname{Var}(\hat{e})}{\operatorname{Var}(Y)}
$$

\footnotetext{
${ }^{1}$ Teker, D., Deniz, E. (2020). Crypto currency applications in financial markets: factors affecting crypto currency prices. Pressacademia, 11, 34-37. DOI: 10.17261/Pressacademia.2020.1235.

${ }^{2}$ Brukhanskyi, R., Spilnyk, I. (2019). Crypto Assets in the System of Accounting and Reporting. The Problems of Economy. 2. 145-156. <http://dx.doi.org/10.32983/2222-0712-2019-2-145-156> (2020, September 17).

${ }^{3}$ Bouri, E., Gupta, R., Lahiani, A., Shahbaz, M. (2018). Testing for asymmetric nonlinear short-and long-run relationships between bitcoin, aggregate commodity and gold prices. Resources Policy, Elsevier, 57, $224-235$. DOI: 10.1016/j.resourpol.2018.03.008.

${ }^{4}$ Tarasova, T., Usatenko, O., Makurin, A., Ivanenko, V \& Cherchata, A. (2020). Accounting and features of mathematical modeling of the system to forecast cryptocurrency exchange rate. Accounting, 6 (3), 357-364.

${ }^{5}$ Yatsyk, T. V. (2018) Methodology of financial accounting of cryptocurrencies according to the ifrs. Evropsky časopis ekonomiky a management, 4 (6), 53-61.

${ }^{6}$ Fisher, R. A. (1925). Theory of statistical estimation. Proc. Cambridge Philos. Soc. 22, 700-725.

${ }^{7}$ Yule, G. U. (1907). On the theory of correlation for any number of variables, treated by a new system of notation.

Proc. Roy. Soc. London ser. A 79, 182-193.

${ }^{8}$ Gauss, C. F. (1809/1963). Theoria Motus Corporum Coelestium. Dover, New York, reprinted 1963.
} 
It is known, that the coefficient of determination is the square of the correlation between predicted $y$ scores and actual y scores, therefore, it ranges between 0 and 1 . If coefficient of determination equals 1 , this means, that the dependent variable can be predicted without an error from the independent variable.

Results and Discussion. Summary statistics of the prices of the currencies are given below.

Table 2

\section{Regression Result (daily closing prices of Bitcoin cash, Bitcoin, Ethereum and other altcoins from 27 December 2013 until 5 January 2020)}

\begin{tabular}{cccccc}
\hline & BCH_P & BITCOIN_P & ETH_P & MyST_P & QUANT_P \\
\hline Mean & 255.2205 & 3626.026 & 151.168 & 0.165071 & 1.072395 \\
Median & 0.000000 & 1054.925 & 17.675 & 0.000000 & 0.000000 \\
Maximum & 3923.07 & 19497.40 & 1396.420 & 3.67000 & 14.29000 \\
Minimum & 0.000000 & 178.1 & 0.000000 & 0.000000 & 0.000000 \\
Std. Dev. & 462.6626 & 3903.032 & 221.1946 & 0.402055 & 2.163755 \\
Skewness & 2.996201 & 0.984969 & 2.189765 & 3.798093 & 2.260604 \\
Kurtosis & 14.31589 & 3.138641 & 8.628193 & 20.56644 & 6.066056 \\
Jarque-Bera & 1581271 & 376.6633 & 4304.17 & 3537672 & 4455.402 \\
Probability & 0.000000 & 0.000000 & 0.000000 & 0.000000 & 0.000000 \\
sum & 591619.7 & 8406988 & 350407.4 & 382.6338 & 2465.813 \\
Sum Sq. Dev. & $4.96 \mathrm{E}+08$ & $3.63 \mathrm{E}+10$ & $1.13 \mathrm{E}+08$ & 374.5991 & 10847.81 \\
Observations & 2318 & 2318 & 2318 & 2318 & 2318 \\
\hline
\end{tabular}

Source: Compiled by the author with Eviews software based on data from Coinmarketcap (2020) ${ }^{1}$

About 2318 observations were performed with Eviews software. From table 2, it can clearly be seen that all the five cryptocurrencies are positively skewed with Bitcoin being the least skewed. In terms of kurtosis, Bitcoin rates are showing less peakedness than a normal distribution, and others are highly peaked than normal distribution with Mysterium showing the maximum peakedness.

Based on the conducted research most cryptocurrencies are affected by external factors such as macroeconomic crises, prohibition on usage of cryptocurrencies in other countries, adoption of restrictive legislation and general supply and demand ${ }^{2}$. Thus, market capitalization of overwhelming majority of different cryptocurrencies has fluctuated due to the COVID-19 pandemic and lockdown as well as due to the increasing digital asset regulations in early 2020 (figure 1). Therefore, during March 2020 the market capitalization of Bitcoin sharply decreased by USD 71,822 million (from USD 166,728 million as at March, 6 to USD 94,906 million as at March, 17). The same trend has a historical change of Bitcoin closing price with a significant drop in March 2020. While most scientists agree, that external factors have considerable effect on cryptocurrencies, there are still very little research on the level of dependence between the prices of Bitcoin and other altcoins. Thus, most cryptocurrencies have a high period around late 2017.

Leitch, Mladenovic (2018) studied the control frameworks for cryptocurrencies at initial evaluation and defined four broad types of cryptocurrency life cycles, such as: "falling away", growing, "flash in the pan" and "flying start" 3 . This taxonomy was based on two main factors: the existence of promotional effort and speed of gain or sustain momentum. The author performed an analysis of different cryptocurrencies using the defined four broad types of cryptocurrency life cycles.

\footnotetext{
${ }^{1}$ Cryptocurrencies market capitalization. Coinmarketcap (2020) <https://coinmarketcap.com/currencies/bitcoin/> (2020, August, 25).

${ }^{2}$ Derun, I., Sklyaruk, I. (2018). The ontological aspects of the essence of cryptocurrency and its display in accounting. Scientific notes of Ostroh Academy National University. Economics series: Scientific journal, 11(39), 163-170.

DOI: http://dx.doi.org/10.25264/2311-5149-2018-11(39)-163-170.

${ }^{3}$ Munro, A. (2020). Cryptocurrency trends in 2020: From DeFi to COVID crisis.

<https://www.finder.com.au/cryptocurrency-trends-in-2020-from-defi-to-covid-crisis> (2020, August, 25).
} 


\section{Cryptoassets' Market Capitalization, USD million (historical)}

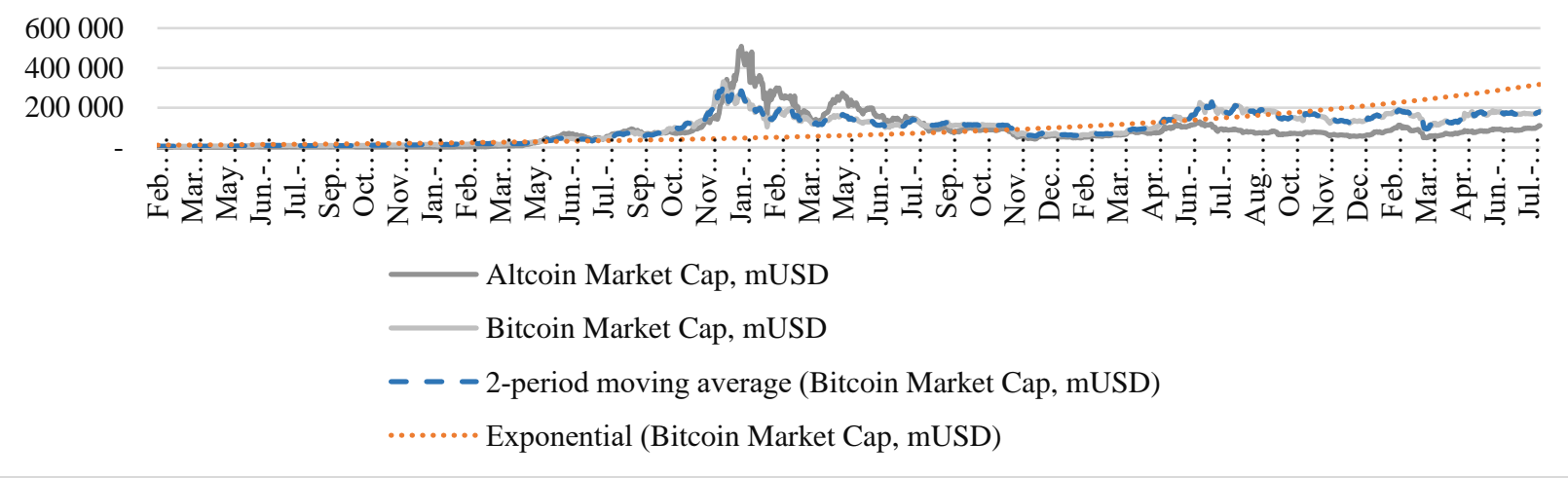

Figure 1. Cryptoassets' Market Capitalization, USD million (historical)

\section{Source: Compiled by the author with Eviews software based on data from Coinmarketcap $(2020)^{1}$}

The most significant and perspective group of cryptocurrencies in terms of current market value and interest for stakeholders are those, that are in the phase of growth or stabilization, because they are the most promising in terms of investment. It contains Bitcoin, Ether, Ripple, Litecoin, Dash and others. Figure 2 shows the exchange rate for Bitcoin. From the figure 2 it could be seen, that there were two high points: the first one at the end of 2013 and a second one at the end of 2017. In between these the price did not fall to its pre-2013 value. Instead, some momentum was sustained allowing another surge later. Other cryptocurrencies in this group show a similar pattern.

The reasons for the rapid rise and fall in price in 2013 are not clear. The FBI's closure of the Silk Road market for contraband had only a tiny effect but the problems at the Mt. Gox exchange may have been more important. The reasons for the halt of the 2017 rise are not yet clear but may be connected with the increased tendency for users to speculate on cryptocurrency prices and the fixed rate of money supply growth.

There are cryptocurrencies that were launched without a significant promotion effort, that never really got started, and quietly disappeared. Also, some launched years ago achieved some distribution but now seem to be losing support.

Linear regression is ruled out since the data is non-random, auto correlated and heteroscedastic in nature ${ }^{2}$. Run test is used to test for randomness of the data: Code, values above the median as positive and values below the median as negative. A run is defined as a series of consecutive positive or negative values.

The first model performed explains the dependencies of Bitcoin price from Google search index and Ethereum price as a second most popular cryptocurrency after Bitcoin. Bitcoin closing price significantly correlated with Google search index. A least-squares multiple regression was estimated using cross-sectional data from 1721 observations after the adjustments, with the following specification:

$$
\log \left(\text { Bitcoin }_{-} p\right)=0.58 * \log \left(\text { Eth }_{-} p\right)+5.46 E \quad 06 * \text { Google_search_I }+5.39
$$

where:

Log (Bitcoin_p) - is the natural logarithm of the Bitcoin-denominated market closing price on August 10, 2020.

Log (Eth_p) - is the natural logarithm of the Ethereum - denominated market closing price on August 10, 2020.

Google_search_I-Google search interest (index), an unbiased sample of our Google search data categorized and focused on search for the term "Bitcoin".

The results for multiple linear regression model are listed below (table 3).

\footnotetext{
${ }^{1}$ Coinmarketcap (2020). Cryptocurrencies market capitalization <https://coinmarketcap.com/currencies/bitcoin/> (2020, August, 25).

${ }^{2}$ Pearson, E. S. (1926). Review of Statistical Methods for Research Workers. Science Progress, 20, 733-734.
} 


\section{Bitcoin Closing Price, USD (historical)}

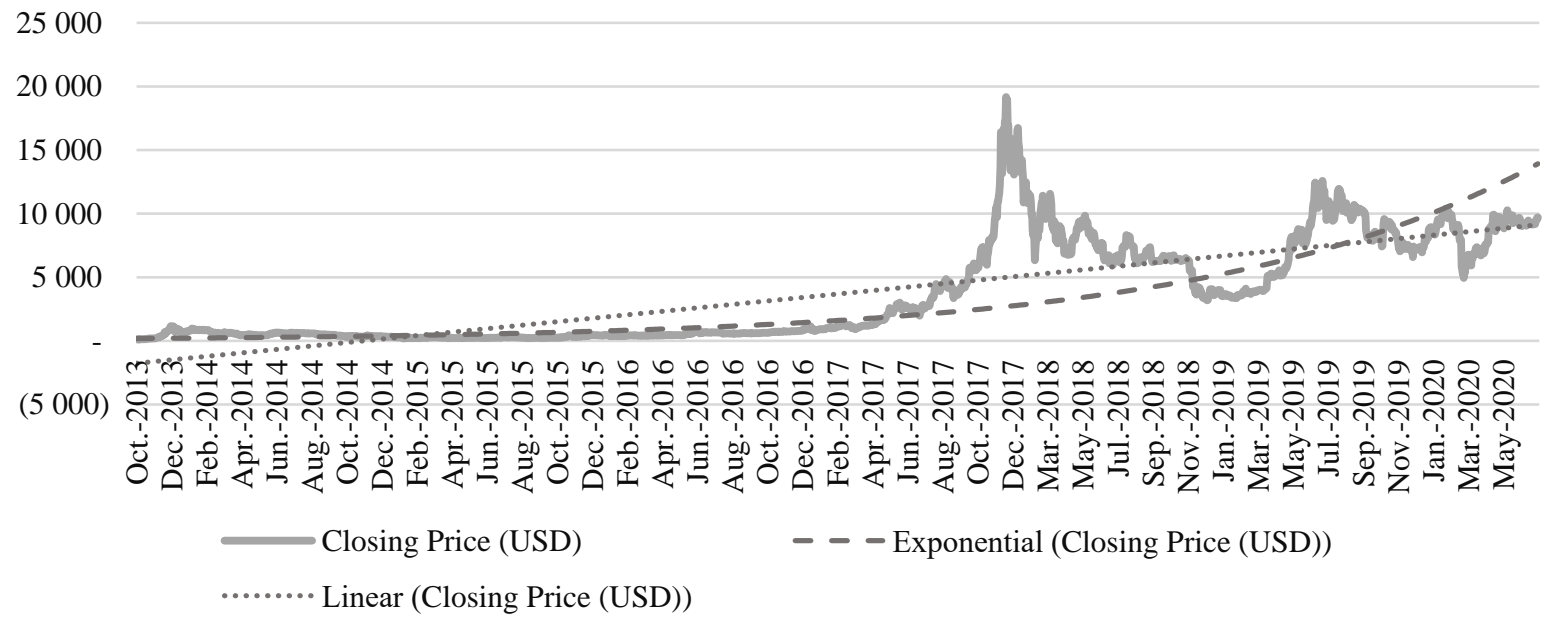

Figure 2. Bitcoin closing price historical

Source: Compiled by the author based on data from ${ }^{1}$.

Table 3

\section{A linear regression model of Bitcoin, Ethereum and Google search index}

Dependent Variable: LOG_BITCOIN_P

Method: Least Squares

Date: 08/10/20 Time: $23: 18$

Sample (adjustedd): 8/16/2015 5/01/2020

Included observations: 1721 after adjustments

\begin{tabular}{lcccc}
\hline \multicolumn{1}{c}{ Variable } & Coefficient & Std. Error & t-Statistic & Prob. \\
\hline \hline C & 5.397703 & 0.0275431 & 212.2527 & 0.0000 \\
LOG_ETH_P & 0.577784 & 0.00621 & 93.03801 & 0.0000 \\
GOOGLE_SEARCH_I & $5.46 E+06$ & $9.69 E+07$ & 5.632111 & 0.0000 \\
\hline \hline R-squared & 0.875478 & Mean dependent var & 7.871050 \\
Adjusted R-squared & 0.875333 & S.D. dependent var & 1.273870 \\
S.E. of regression & 0.449782 & Akaike info criterion & 1.241633 \\
Sum squared resid & 347.5574 & Schware criterion & 1.251134 \\
Log likelihood & -1065.424 & Hannan-Guinn criter. & 1.245146 \\
F-statistic & 6039.353 & Durbin-Watson stat & 0.007579 \\
Prob (F-statistic) & 0.0000 & & & \\
\hline \hline
\end{tabular}

Source: Compiled by the author based on data from Coinmarketcap (2020) with Eviews software.

The R2 is quite high, suggesting that approximately $87.54 \%$ of the variation in relative cryptocurrency prices are determined by the variables in the model. The coefficients for variables are positive, thus the price increase as search interest increase, as well as increase of Ethereum price. The t-statistic indicates that it is highly statistically significant that google search interest influences Bitcoin price.

${ }^{1}$ Coinmarketcap (2020). Cryptocurrencies market capitalization <https://coinmarketcap.com/currencies/bitcoin/> (2020, August, 25). 
Another model was performed to analyse the dependencies between Bitcoin closing price and closing price of other altcoins (table 4).

Least squares multiple regression was estimated with the following specification:

$$
\log \left(\text { Bitcoin } \_p\right)=0.50 * \log (\text { Eth_p })+1.34 E \quad 05 * \text { Google_search_I }+0.006 * \text { Num }_{-} p+0.13^{*} \text { Quant_p } 0.22 * \text { Myst_p }+5.43
$$

Table 4

\section{A linear regression model of Bitcoin, Ethereum and Google search index}

Dependent Variable: LOG_BITCOIN_P

Method: Least Squares

Date: 08/15/20 Time: 19:43

Sample (adjustedd): 8/16/2015 5/01/2020

Included observations: 1721 after adjustments

\begin{tabular}{lcccc}
\hline \multicolumn{1}{c}{ Variable } & Coefficient & Std. Error & t-Statistic & Prob. \\
\hline \hline C & 5.430412 & 0.018306 & 296.643 & 0.0000 \\
LOG_ETH_P & 0.506069 & 0.00534 & 94.76293 & 0.0000 \\
GOOGLE_SEARCH_I & & & 17.9975 & 0.0000 \\
NUM_P & 0.006797 & 0.001462 & 4.645881 & 0.0000 \\
QUANT_P & 0.134924 & 0.003658 & 36.88353 & 0.0000 \\
MYST_P & -0.224176 & 0.030483 & -7.354238 & 0.0000 \\
\hline \hline R-squared & 0.940125 & Mean dependent var & 7.871050 \\
Adjusted R-squared & 0.93995 & S.D. dependent var & 1.273870 \\
S.E. of regression & 0.312163 & Akaike info criterion & 0.512899 \\
Sum squared resid & 167.1196 & Schware criterion & 0.531902 \\
Log likelihood & -435.3493 & Hannan-Guinn criter. & 0.519929 \\
F-statistic & 5385.562 & Durbin-Watson stat & 0.034261 \\
Prob (F-statistic) & 0.0000 & & \\
\hline \hline
\end{tabular}

Source: Compiled by the author based on data from Coinmarketcap (2020) with Eviews software.

where:

MYST_P - daily closing prices for cryptocurrency Mysterium.

QUANT_P -daily closing prices for cryptocurrency Quant.

NUM_P - daily closing prices for cryptocurrency Numeraire.

Similar to the previous analysis of the primary model, all coefficients are significant, as their Probability is less than 0.05 . To test the model for adequacy, it is necessary to compare the values of F-statistics with the theoretical value of Fisher's statistics. For this model, the practical value is F-statistic $=5385$.

Conclusions. On the basis of the above overview and the above analysis a regression model can be used for predicting the exchange rate of Bitcoin. A cryptocurrency is a digital or virtual currency designed to work as a medium of exchange. It uses cryptography to secure and verify transactions as well as to control the creation of new units of a particular cryptocurrency. The COVID-19 pandemic has paralyzed, disrupted and stopped many industries and made numerous governments think about remote work, cashless economy, distributed ledger technology and possibility to use cryptoassets. The number of Internet users around the world has more than doubled over the last decade. Decentralized finance (DeFi) has become one of the most prominent trends in crypto since late 2019. Thus, 2020 is going to be called the best year to invest in cryptocurrencies.

Data used in this paper for the regression analysis contains historical data related to closing cryptocurrency prices, Google search index, transaction volume, capitalization etc. Five important cryptocurrencies with different life cycles were analysed and used in multiple linear regression model for cryptocurrency price (Bitcoin cash, Bitcoin, Ethereum, Mysterium, Quant). Based on the conducted 
research most cryptocurrencies are affected by external factors such as macroeconomic crises, prohibition on usage of cryptocurrencies in other countries, adoption of restrictive legislation and general supply and demand. However, when discussing alternative finance, it is impossible to ignore the current economic crisis or to understate its potential impact on cryptocurrency.

\section{References:}

1. EY (2019). IFRS Developments: Holdings of cryptocurrencies <https://www.ey.com/Publication/vwLUAssets/ ey-devel150-cryptocurrency-holdings-august-2019/USDFILE/ey-devel150-cryptocurrency-holdings-august-2019.pdf> (2020, August, 25). [in English].

2. Ankenbrand, T., Bieri, D., Cortivo, R., Hoehener, J., Hardjono T., (2020). Proposal for a Comprehensive (Crypto) Asset Taxonomy. 2020 Crypto Valley Conference on Blockchain Technology (CVCBT), Rotkreuz, Switzerland, 16-26. [in English].

3. Coinmarketcap (2020). Cryptocurrencies market capitalization. <https://coinmarketcap.com/currencies/bitcoin/> (2020, August, 25). [in English].

4. Coindesk (2020). Bitcoin market capitalization <https://www.coindesk.com/price/bitcoin> (2020, August, 25) [in English].

5. Financial Conduct Authority (FCA) (2019). Guidance on Cryptoassets. Consultation Paper CP19/3 <https://www.fca.org.uk/publication/consultation/cp19-03.pdf> (2020, September 17). [in English].

6. Akbulaev, N., Mammadov, I., Hemdullayeva, M. (2020). Correlation and Regression Analysis of the Relation between Ethereum Price and Both Its Volume and Bitcoin Price. The Journal of Structured Finance. DOI: jsf.2020.1.099. 10.3905/jsf.2020.1.099. [in English].

7. Teker, D., Deniz, E. (2020). Crypto currency applications in financial markets: factors affecting crypto currency prices. Pressacademia, 11, 34-37. DOI: 10.17261/Pressacademia.2020.1235 [in English].

8. Brukhanskyi, R., Spilnyk, I. (2019). Crypto Assets in the System of Accounting and Reporting. The Problems of Economy, 2. 145-156. DOI: http://dx.doi.org/10.32983/2222-0712-2019-2-145-156> (2020, September 17) [in English].

9. Bouri, E., Gupta, R., Lahiani, A., Shahbaz, M. (2018). Testing for asymmetric nonlinear short-and long-run relationships between bitcoin, aggregate commodity and gold prices. Resources Policy, Elsevier, 57, 224-235. DOI: 10.1016/j.resourpol.2018.03.008. [in English].

10. Tarasova, T., Usatenko, O., Makurin, A., Ivanenko, V., Cherchata, A. (2020). Accounting and features of mathematical modeling of the system to forecast cryptocurrency exchange rate. Accounting, 6(3), 357-364. [in English].

11. Yatsyk, T. V. (2018) Methodology of financial accounting of cryptocurrencies according to the ifrs. European Journal of Economics and Management, 4 (6), 53-61 [in English].

12. Fisher, R. A. (1925). Theory of statistical estimation. Proc. Cambridge Philos. Soc, 22, 700-725. [in English].

13. Yule, G. U. (1907). On the theory of correlation for any number of variables, treated by a new system of notation. Proc. Roy. Soc. London ser. A, 79, 182-193. [in English].

14. Gauss, C. F. (1809/1963). Theoria Motus Corporum Coelestium. Dover, New York, reprinted 1963 [in English].

15. Derun, I., Sklyaruk, I. (2018). The ontological aspects of the essence of cryptocurrency and its display in accounting. Scientific notes of Ostroh Academy National University. Economics series: Scientific journal, 11(39), 163-170. DOI: http://dx.doi.org/10.25264/2311-5149-2018-11(39)-163-170. [in English].

16. Munro, A. (2020). Cryptocurrency trends in 2020: From DeFi to COVID crisis. Finder AU $<$ https://www.finder.com.au/cryptocurrency-trends-in-2020-from-defi-to-covid-crisis> (2020, August, 25). [in English].

17. Pearson, E. S. (1926). Review of Statistical Methods for Research Workers, by R. A. Fisher. Science Progress, 20 , 733-734. [in English]. 